\title{
Making Profitable GNSS RTK Network Infrastructure
}

\author{
J. van Cranenbroek, Vincent Lui* and Ryan Keenan. \\ Networked Reference Stations and Structural Monitoring \\ Leica Geosystems, Heinrich-Wild-Strasse, Heerbrugg, 9435, Switzerland \\ *Leica Greater China GSR \\ *Leica Geosystems Ltd, 25 Westlands Road, Quarry Bay, Hong Kong
}

constellations like GALILEO will just amplify this trend that will capitalize on the accuracy, reliability and highspeed location addicts.

Keywords. GPS, Network, RTK, corrections, business, profit, services.

\section{Introduction}

It is well recognized today that a reference network comprised of permanent GPS stations provides the fundamental infrastructure required to meet the needs of professional GPS users in many areas of surveying and mapping. Examples of applications are survey control work, densification of existing geodetic networks, acquisition of data for GIS applications, cadastral operations, determination of fiducial points for photogrammetric work, monitoring of engineering works, mapping of utility corridors and assets, etc. In fact, the number of applications benefiting from the establishment of permanent networks seems to be growing daily.

The widespread use of RTK GPS and DGPS techniques has encouraged decision-makers to look for ways to replace traditional geodetic networks with networks of permanent GPS reference stations. For example, a tighter control of the networks can be achieved from the data supplied by permanent reference stations both in postprocessing and in real-time. With streaming data, the influence of those spatial and temporal errors affecting GPS measurements can be estimated in real-time. This in turn means the quality of the transmitted corrections is improved and the range of RTK GPS increased.

Since 2001, Leica Geosystems has promoted the Master Auxiliary concept to increase the performance and throughput of permanent networks. Additionally, discussions taking place within the RTCM organization 
aim to introduce new standards for the broadcast of GPS corrections specifically for Network RTK. Leica has been actively involved with the working group responsible for this message standardization.

Recently Leica Geosystems has released its GPS SpiderNET software, implemented with several innovative mechanisms such as network clustering, auto cell generator and individualized network corrections.

In the following discussion we will attempt to gain a better understanding of what has now become a fundamental question: how to convince decision-makers to commit adequate resources to realise their projects, and how do the new transaction modes meet the needs of their end users?

\section{Can the Costs Associated with GNSS RTK Network be Recovered?}

The majority of permanent GPS networks has been, and will continue to be, for some time to come, initiatives primarily from government agencies.

These government entities have been able to justify the costs of implementing GPS networks by citing the approach of "preventable costs"; similar to the strategy used to finance the establishment of classical geodetic networks decades earlier. The return on the original investment is not measured in nominal terms of hard revenue but in keeping the costs borne by the industry lower than the alternative (i.e. no geodetic infrastructure). This approach also encourages network standardization and avoids the appearance of a patchwork of private, customized networks for project-specific purposes.

The net result of these free, but limited, services has been to give the user the impression that the distribution of differential GPS corrections should remain free of charge, and that the cost of establishing and maintaining the networks, and providing services, should be assumed by the network operators.

This observation is supported by the marked decrease in the number of paying users for the GPS correction services provided by companies such as DCI, and even Omnistar just a few years ago. They have since struggled to remain competitive in the face of the U.S. presidential decision to turn off Selective Availability on the GPS signals.

Even today, many agencies are facing an uphill battle in trying to convince potential users to subscribe to their GPS correction services. The primary reason is the disproportionate cost for the offered services when borne by a limited number of customers.

Given the prevailing attitude, Leica Geosystems must find ways to justify investment of substantial resources in
R\&D. In particular, we must be able to convince our future customers that they will see a return on their investment, not only in a positive practical sense, but that they may even financially profit from it.

It may be useful to compare our present situation with that of cellular phone service providers several years ago. There is no denying that these companies are now seeing healthy profits from the various levels of wireless service they offer today. However, when the products were first introduced to the public the companies gambled on the presupposed reliability and variety of services to lure the customers, and offset an often complex and costly infrastructure. Evidence that their investments paid off can be found in the steady increase in the number of users over the years and the attraction of new service offerings being rolled out on a regular basis. These services are indeed new applications that users have been willing to pay for. Our numerous and ongoing meetings with potential customers have led us to re-think our approach and put together a new vision.

\section{A New Information Broadcast Service Based on GPS Network}

This is not wishful thinking resulting from our infatuation with communication technologies, but truly an achievable goal. Decision-makers wish to control the quality of services based on the type of products their networks provide.

They are also committed to providing GPS network solutions in the appropriate reference system. Coordinate transformations should not been seen purely as side products: the very purpose of permanent GPS networks is to offer a complete integrated datum-consistent solution including geoidal height correction. To those who argue that the transformation algorithms could be integrated into the rover units, and that a certain level of control can be achieved by forcing the user to calibrate his system on existing control points; we answer that in doing so, we have eschewed our responsibility for providing a complete solution.

We must add also that the software applications used to manage permanent GPS networks could, and even should, incorporate functions to monitor usage and/or charge users for services.

Leica Geosystems has already begun to integrate usermonitoring applications into its GPS SpiderNET software by including the display of user positions, recording the number of requests for specific services, and generating statistical information that forms the basis for charging users.

The GPS RTK Network owners, and potential owners, now consider this type of functionality essential, and we 
are hearing more and more requests from customers interested in software utilities giving them the ability to exercise control over their networks in order to enhance the value of their services.

The subject of increased data integrity is also creating considerable interest among GPS network operators. What if they could provide a service that bypassed the problems that users routinely encounter in processing their own data?

A reliable network service providing high quality and high fidelity solutions would no doubt generate significant revenue. It is clear that most of the GPS RTK Network operators today hesitate to generate profit by charging for their services only because they cannot provide continuous and reliable services. In many cases these organizations have their initial background in surveying and geodesy, and few of them have the IT specialists, and even the resources, to handle a decent service. In addition there is a lack of marketing and promotional effort, as well as a reluctance to target new customers and niche applications.

\section{A Few Crucial Questions}

It is clear that still today most of the GPS RTK Network operators are looking much more to technology performance and on the possibility to provide an economic means of improving the use of GPS RTK surveying, for example by allowing users to perform longer baselines with still a high and homogeneous accuracy.

Few of them are designing their infrastructure with an appropriate benefit-cost analysis that would allow them to compare the expected cost of implementing a system with the expected benefits that will result from having the new correction/data product. The result of this comparison is generally a good indication of whether or not a project would be financially viable, and when the operator could expect an actual financial return on his initial investment.

In many cases, the preference goes to deploy a dense and large network covering systematically the complete area regardless of the existing or potential surveying activity. The second generation of GPS RTK Network software allows however a much more flexible and scalable approach. We can setup a GPS RTK Network today sequentially by phasing the whole project.

And today, even after a successful installation, the responsible of the infrastructure starts only at that time when contact is made with other organizations and potential user groups to attract them to their services. As the existing users have been trained to deploy their own base station, they may not be willing to pay for a GPS
RTK service that they can support themselves. The apparent difficulty to turn this kind of high tech infrastructure into profitable business seems also to be due to the fact that the actual service providers are in a direct relationship with their users.

For managing such a service, the network operator should delegate the business part of his job to a professional company, which has a strong financial situation, and good experience in developing new services and is capable of implementing a charging mechanism. But those new business operators will want to have the technology able to support their business model.

\section{Client-Server Approach}

How about if, instead of broadcasting corrections and placing the onus of obtaining a final solution on the user and his equipment, we took advantage of our existing network system infrastructure to compute his coordinates pre-broadcast in the required reference system? Final (position) solutions for all logged users would be simply computed as a by-product of the continuous network processes - all the time satisfying the quality and integrity criteria implemented at the network administrator level. After all, there exist already a number of web-based services for the generation of coordinates via the post-processing of data submitted by the user. We are proposing to extend this functionality to real-time processing.

This proposal is certainly worth investigating! Currently, providers of GPS corrections have no control over the quality of the results computed by the user. This makes it difficult for them to justify charging for their services.

Compounding the problem, providers of GPS hardware typically implement their own proprietary algorithms to compute an RTK-derived position. For instance, a GPS Network RTK software provider has introduced the concept of Pseudo Reference Station, which works by purposefully exaggerating the distance of a so-called Virtual Reference Station to "force" rover algorithms biased by the user's proximity to an existing station to apply ionospheric and tropospheric corrections.

In other words, the very concept of virtual reference stations has been spawned by the fact that rover-based RTK solutions exhibit inherent and historic biases, particularly with distance-dependency. This has led to the development of many solution algorithms to tackle this problem. The current approach proposed by the RTCM standards committee will only compound the difficulties by forcing the burden of computational work onto the rover firmware. Overall this situation leaves providers in a weakened position to charge for their services since 
they do not have any control over the quality of the solutions generated in the field using their data.

Therefore, we believe that a paradigm shift is required in order to meet the expectations of future network operators.

A "Client-Server" approach contradicts the data flow in conventional RTK by asking the field user to transmit his data to the control center. This facility can select the optimal combination of stations to apply network corrections and compute the best possible position before returning the result to the user.

Clearly, decision-makers meet the nominal premise of delivering a homogeneous solution to all users regardless of their GPS hardware with increasing interest because it addresses many of their concerns with respect to profitability.

The advantages of this approach are clearly evident. We can exercise control over the generated products and, as a result, place a commercial value on the service, especially as the typical user is released from the obligation of learning complicated GPS surveying techniques. Safeguards, and thus integrity, can also be easily implemented into the distribution service; if the number of satellites is too low, the geometry unfavorable, or the multipath effects too detrimental, a message can be sent back to the user warning them that the provided solution is not optimal and that it may not meet their specifications. With the critical processes ("legal minefields") of traceability and integrity looming on the horizon for positioning services, these grey areas are becoming much 'darker' and need to be considered in any long-term plans by all software providers involved with GPS positioning.

An added benefit to this approach is the decreased burden placed on the rovers by removing the need for field calculations, thus encouraging the development of a new generation of less costly rovers. These 'no-frills' (stripped-down) receivers are already beginning to hit the market.

\section{Conclusions}

The key to a profitable GPS RTK Network lies first to the implementation of several good practice habits that include benefit-cost analysis, market research and a stepby-step deployment approach.

However, the way such infrastructures are used to generate the corrections plays also a role in the development of a profitable product. With the trend towards lower cost GPS equipment, it is clear that putting the computational effort on the server side will justify more easily the charging the users for a value-added product: it's the user's accurate position in the local reference frame.

We believe that the second generation of GPS RTK Network processing software will contribute significantly to the deployment of more profitable GPS infrastructure.

\section{References}

van Cranenbroeck J. (2004) GPS Network Services for Supporting Surveying Tasks, Map of India New Delhi, India.

www.gisdevelopment.net/technology/survey/mi04150.htm

Leica Geosystems (2005) An introduction to the philosophy and technology behind Leica Geosystems' SpiderNET revolutionary Network RTK software and algorithms. Leica Geosystems AG, Heerbrugg, Switzerland, June 2005. www.leicageosystems.com/common/shared/downloads/inc/download er.asp?id $=5367$ 